\title{
Fabrication of Organic Solar Cells with Branched Cauliflower-Like Nano Structures as a Back Electrode Replicated from a Natural Template of Cicada Wing Patterns
}

\author{
Hamzeh Nourolahi $^{\mathrm{a}}$, Mohammad Agha Bolorizadeha ${ }^{\mathrm{a}}$, and Abbas Behjat ${ }^{\mathrm{b}, \mathrm{c}, *}$ \\ ${ }^{a}$ Photonics Research Group, Research Institute of Science and High Technology and the \\ Environmental Sciences, Graduate University of Advanced Technology, Kerman, Iran \\ ${ }^{b}$ Photonics Research Group, Engineering Research Centre, Yazd University, Yazd, Iran \\ ${ }^{c}$ Atomic and Molecular Groups, Faculty of Physics, Yazd University, Yazd, Iran \\ *Corresponding Author's Email: abehjat@yazd.ac.ir
}

Received: Nov. 4, 2015, Revised: Mar. 3, 2016, Accepted: Jul. 27, 2016, Available Online: Jan. 28, 2017

DOI: 10.18869 /acadpub.ijop.11.1.39

\begin{abstract}
Nanostructures of noble metal materials have been used in organic solar cells for enhancement of performance and light trapping. In this study, we have introduced branched silver cauliflower-like nanopatterns as sub-wavelength structured metal grating in organic solar cells. Self-assembled fabrication process of branched nanopatterns was carried out on a bio-template of cicada wing nanonipple arrays using a gas aggregation dc magnetron sputtering nanocluster source without size filtration. The branched nanostructures provide surface gaps with dimensions near the organic exciton diffusion length, which prevents recombination of charge carriers. An increased power conversion efficiency of $14.8 \%$ compared to that of the planar device was achieved mainly due to the enhancement in the short-circuit current density. Besides, these branched cauliflower-like nanopatterns had enhanced optical light absorption in the solar cell as a result of enhancing the optical path length of the reflected light in the active layer and plasmonic effects of the noble metal material.
\end{abstract}

KEYWORDS: Organic solar cells, Plasmonics, Cauliflower-like, Nanopattern.

\section{INTRODUCTION}

In recent years, organic solar cells (OSCs) have been a subject of increasing research interest due to their potential of being low cost, large area of production and device flexibility [1-7]. An effective approach to enhance OSCs is to increase the light absorption of the organic active layer without increasing its thickness. Several light trapping strategies have been used to enhance the photon density in OSCs including use of diffraction nano-gratings $[3,8]$, photonic crystals [5], and plasmonic nanostructures [6, 7]. Among these approaches, using the plasmonic nanostructures of noble metal materials for light trapping is quite promising. Physically, plasmonic subwavelength structures of noble metal materials interact with incident light and, thus, can modify the incident wave momentum in such a way that the light remains trapped in the solar cell layers $[8,9]$.

Recently, nanostructured (NS) materials of a particular morphology have attracted broad attention in solar cell research. As one of the most important nanomaterials, branched NS materials have been widely applied in organic and hybrid solar cells [10-13]. In this regard, branched metal NS materials are of specific interest that arises from their unique surfacesensitive properties, large number of multiple surface edges and large surface-to-volume ratio [14-17] . It would be also feasible to tune 
the fascinating properties of branched structures such as their optical and electrical properties by size and shape modifications [18, 19].

Template-based nanostructuring is one of the most promising nanometer-sized patterning techniques in OSCs $[2,3,8,20,21]$. Biotemplates have inspired scientists to make use of natural nanostructures and utilize their especial properties $[20,22,23]$. Natural surface nanostructures of cicada wing exhibit particular optical properties that originate from its specific surface geometry. Cicada wing including quasi-ordered surface nano-nipple arrays has been used as a replicable natural template $[20,22,23]$. Cicada wing has been employed as an antireflective and easily separable substrate from a deposited layer as a result of its low surface tension material that can serve naturally as an anti-sticking coating $[22,24,25]$, and also as a light harvesting in photovoltaic devices [26].

In this study, we have explored a practical method to produce branched silver (Ag) nanocauliflower arrays on the natural template of cicada wing patterns. By using a gas aggregation nanocluster source through $\mathrm{dc}$ magnetron sputtering, the nano-cauliflower arrays were fabricated. This high production volume scheme is similar to the laser vaporization technique $[10,27]$. We separated the deposited Ag layer, including branched cauliflowers like arrays, from the cicada wing template and utilized it as a nanostructured metallic grating back electrode in OSCs. The device structure was Ag cauliflower-like nanopatterned/PEDOT: PSS/P3HT: PCBM/Ag (semitransparent metal electrode). We demonstrated that an enhancement in optical absorption resulting from improvement of light trapping in the active layer increases the short-circuit current density by employing the branched structures on the back contact. Moreover, introducing of these nanocauliflower arrays on the back electrode reveals efficient capture and separation of excitons due to the existence of abundant branches on the electrode. This provides preferential carrier pathways, reducing charge recombination and facilitating carrier collection in OSCs. Consequently, the power conversion efficiency (PCE) of OSCs with branched cauliflower-shaped nanostructure arrays is considerably improved.

\section{EXPERIMENTAL DETAILS}

\section{A. Preparation of branched cauliflower-like nanopatterns}

The cicada's wing substrates (Cryptympana atrata Fabricius) that had quasi-ordered nanonipple arrays on their surface were cut in $5 \times 5 \mathrm{~mm}^{2}$ surface areas and cleaned before use. To remove contaminants absorbed physically on the surface, the samples were sonicated in deionized water for about 10 minutes. The samples were then sonicated in acetone for 30 minutes to remove the stains that might stick the wing's nanonipples together. Finally, to remove residual acetone, the samples were sonicated in deionized water again for 20 minutes and subsequently dried at room temperature. Deposition of silver nanoparticles (NPs) was carried out based on the gas aggregation procedure using the dc magnetron sputtering technique by a commercially available system (DSR1, Nanostructured Coating Co.). The deposition process took place in a vacuum chamber, and the substrates were fixed at $90 \mathrm{~mm}$ from a dc planar magnetron ( $80 \mathrm{~mm}$ in diameter), perpendicular to the cluster source axis.

Ag nanoclusters produced by silver target in the magnetron sputtering source were operated at room temperature. The base pressure in the deposition chamber was $\approx 7 \times 10^{-6}$ mbar. During magnetron sputtering with pure Ar gas, the pressure of $17.3 \times 10^{-2}$ mbar was applied. The discharge power during sputtering was $175 \mathrm{~W}$, and the Ar flow rate was regulated by means of a mass flow controller (MKS Instruments) and fixed at $55 \mathrm{sccm}$ (standard cubic centimeter/minute).

\section{B. Fabrication and characterization of OSCs}

The OSCs were fabricated on both flat glass substrate silver-coated and the branched silver cauliflower-like arrays. The cicada wings with 
a surface area of $5 \times 5 \mathrm{~mm}^{2}$ were adhered to a supporting plate with an organic glue (EPOTEK, Epoxy Technology Inc.). The deposition process of the Ag film was carried out as described in section 2.1.

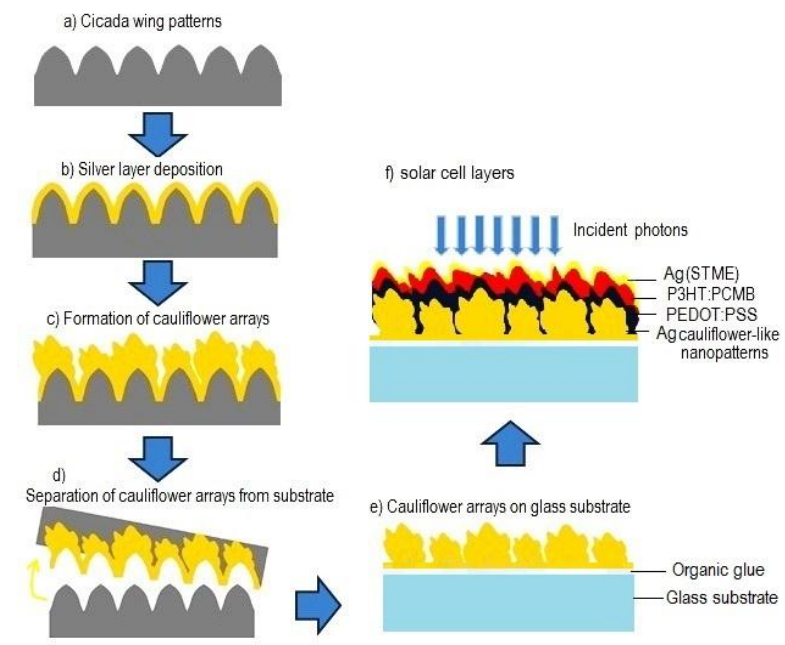

Fig. 1. Schematic diagram of the fabrication procedure of OSC with cauliflower-like nanostructure arrays, using the cicada wing as a biotemplate. The specification of the fabricating steps (a)-(e) for the back electrode layer and (f) for the spin-coated layers and semitransparent metal electrode (STME) deposited on the solar cell in the scheme.

Then, a tape (Scotch Magic Tape, 104-NA) was adhered onto the top side of the cauliflower-like nanopatterned film, and the deposited film was separated mechanically with small tweezers while keeping the wing's nanonipple array structures intact (Fig. 1 (d)). The deposited $\mathrm{Ag}$ layer, or the branched cauliflower-like arrays, was separated from the cicada wing substrate as described schematically in Fig 1. The next step is to transfer the Ag film to the glass substrate (Fig. 1(e)) and, after the glue was dried at room temperature, the scotch tape was separated with a pair of small tweezers to fabricate a back electrode including the branched cauliflower-like NS arrays.

After the fabrication of back electrodes, PEDOT: PSS was spin-coated (3000 rpm) on both sample substrates with the silver-coated flat glass substrate and the branched silver cauliflower-like arrays. Then, it was dried at $120{ }^{\circ} \mathrm{C}$ for 15 minutes. A P3HT: PCBM active layer was deposited by spin-coating from a chlorobenzene solution of P3HT (RMI-001E, Electronic grade, Rieke Metals Inc.) and PCBM (99\%, SES Research) mixed in a 1:0.8 weight ratio at a spin rate of $700 \mathrm{rpm}$ for 30 seconds and dried in a glove box filled with argon gas. Finally, a 10-nm-thick silver piece was deposited under $13 \times 10^{-2}$ mbar pressure by dc magnetron sputtering through a shadow mask to fabricate a semitransparent metal cathode electrode. In this way, a direct comparison between a structured cell and a planar cell was possible making use of similar deposited metal electrodes.

The optical absorption of solar cells was analyzed from the diffuse reflection $(R)$ and the transmission $(\mathrm{T})$ spectra using 1-R-T by an Ultraviolet-visible (UV-Vis) spectrometer system (HR 4000, Ocean Optics). The currentvoltage $(\mathrm{J}-\mathrm{V})$ characteristics were measured by a Keithley 2400 source meter under 1 sun (100 $\mathrm{mW} / \mathrm{cm}^{2}$ ) simulated AM 1.5-G illumination with a solar light simulator $(300 \mathrm{~W}$, Xenon lamp) calibrated by a reference Si solar cell. The surface morphology of the nanostructures was monitored using scanning electron microscope (SEM) images using the TESCAN SEM system. The AFM image was recorded in air using a DME dual scope (DS 95-50) instrument operated in the AC mode. The analysis of the AFM image was performed by using the software provided by this AFM system.

\section{RESULTS AND DISCUSSION}

\section{A. Fabrication of the branched cauliflower- like arrays}

Scanning electron microscope (SEM) images of the cicada wing top view $\left(\mathrm{C}_{0}\right)$ and tilted view $\left(C_{1}\right)$ showed the structures of nanonipple arrays using the TESCAN SEM system (Fig. 1). The height of the nanonipples with an average diameter of $70 \mathrm{~nm}$ (in $\mathrm{C}_{0}$ and $\mathrm{C}_{1}$ ) was about $300 \mathrm{~nm}$, and their spacing was around $130 \mathrm{~nm}$. Three samples (i.e. cicada wing /Ag with three different operational conditions) were fabricated to illustrate the effect of operational conditions of $\mathrm{dc}$ magnetron 
sputtering on the formation of NS arrays onto nanonipple arrays.
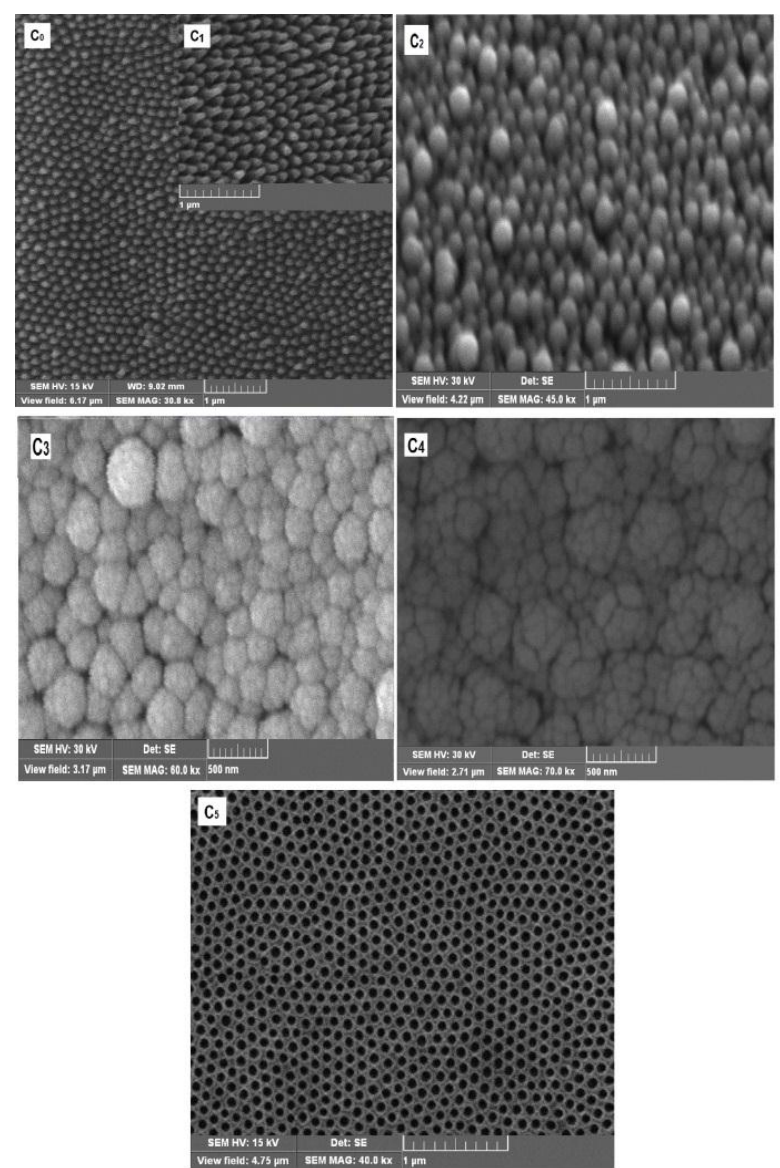

Fig. 2. SEM images of the cicada wing top view $\left(C_{0}\right)$ and tilted view $\left(C_{1}\right)$ showing the structures of nanonipple arrays; the surface structures of the $\mathrm{Ag}$ nanostructures obtained in different operational conditions of $\mathrm{dc}$ magnetron sputtering $\left(\mathrm{C}_{2}, \mathrm{C}_{3}\right.$, and $\mathrm{C}_{4}$ ); the back side of nanocauliflower arrays, the structure of quasi-ordered nano-hole arrays $\left(\mathrm{C}_{5}\right)$ imprinted from nanonipple arrays onto the silver deposited layer.

In the aggregation chamber, different conditions of Ar gas pressure and discharge power were applied while other parameters such as distance from magnetron source and gas temperature were kept constant. There emerged a 3D silver branched nanocauliflower-like structure. The surface morphology of the Ag NS obtained in different operational conditions $\left(\mathrm{C}_{2}, \mathrm{C}_{3}\right.$, and $\left.\mathrm{C}_{4}\right)$ is shown in Fig. 2. The Ag nanocauliflowers on sample $\mathrm{C}_{4}$ have surface edges and a branched surface that is arranged on the substrate largely with an upward head direction. These nanocauliflowers show varying dimensions in the range of $200-450 \mathrm{~nm}$, and have surface gaps with dimensions between 10 to $35 \mathrm{~nm}$. Some cauliflowers having a larger size are observed further up, and some smaller ones are also produced and found to be located beneath the larger aggregates.
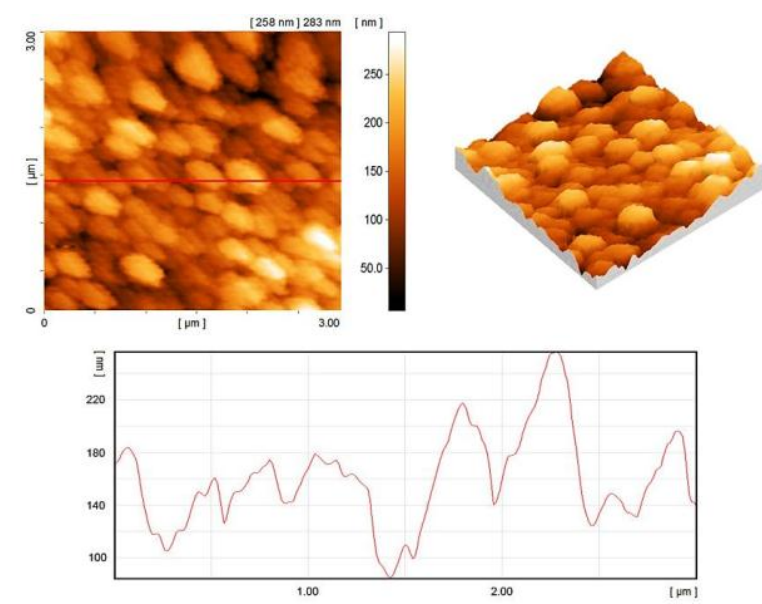

Fig. 3. AFM surface morphology of the solar cell back electrode including nanocauliflower arrays spin-coated by PEDOT: PSS layer, 3D image of AFM topography analysis (Top right), 2D surface morphology (top left), and corresponding profile of the surface morphology (down).

In sample $\mathrm{C}_{5}$, namely the back side of the nanocauliflower arrays, one can observe the structure of the quasi-ordered nano-hole arrays imprinted onto the silver layer deposited from the nanonipple arrays of the substrate template. The nano-holes have diameters of around $70 \mathrm{~nm}$ and an average pitch of about $130 \mathrm{~nm}$. Atomic force microscopy (AFM) analysis was carried out to accurately study the surface morphology of the solar cell back electrode including nanocauliflower-like surface features spin-coated by PEDOT: PSS layer. In the 2D and 3D AFM morphology analysis, as shown in top left and right parts Fig. 3, the nanocauliflower arrays on the back electrode are completely transferred to PEDOT:PSS spin-coated layer.

Different operational conditions of the gas aggregation chamber and the discharge power for fabrication of samples $\mathrm{C}_{2}, \mathrm{C}_{3}$, and $\mathrm{C}_{4}$ are given in Table 1. Increasing the discharge power usually increases the mean energy of $\mathrm{Ar}^{+}$ions in the aggregation chamber, and, therefore, the amount of sputtering atoms 
increases [28]. Ar serves as the buffer gas for the cluster formation and is responsible for sputtering. An increase of the gas pressure due to increasing of Ar flow rate leads to a slight increase of the cluster size [29].

Table 1. Operational conditions of the gas aggregation chamber and the discharge power for fabrication of samples $\mathrm{C}_{2}, \mathrm{C}_{3}$, and $\mathrm{C}_{4}$

\begin{tabular}{|c|c|c|c|}
\hline $\begin{array}{l}\text { Operational } \\
\text { condition }\end{array}$ & Sample $\mathrm{C}_{2}$ & Sample $C_{3}$ & Sample $\mathrm{C}_{4}$ \\
\hline $\begin{array}{l}\text { Ar Gas } \\
\text { pressure } \\
\text { (mbar) }\end{array}$ & $12.8 \times 10^{-2}$ & $14.6 \times 10^{-2}$ & $17.3 \times 10^{-2}$ \\
\hline $\begin{array}{l}\text { Discharge } \\
\text { Power (W) }\end{array}$ & 120 & 140 & 175 \\
\hline
\end{tabular}

It is evident that an increase in the gas pressure leads either to a shorter aggregation time and a longer cluster size or to an increase in the deposition rate of atoms. The charge on the sharp surface edges on the substrate that have grown up with the initial $\mathrm{Ag}$ particles is presumed to repel the subsequent incident particles by Coulomb force, and the following particles deposit on a place that has a lower electric repulsion force, hence creating aggregates with gaps between them and forming branched cauliflower-like structures. Due to the increase in the deposition rate which is caused by a change in the Ar gas pressure and the discharge power in the aggregation chamber, the branches appear on nano-arrays and start to form cauliflower-like geometry feature.

The cicada wing properties are suitable for separating of the deposited film from its surface while keeping the wing's nanonipple array structures intact. The main element of the cicada wing template is a high molecular weight; the crystalline polymer-whose Young's modulus is as high as 7-9 GPa [25], which is high enough to conserve the original wing surface structure during the separation process of the deposited film including nanocauliflower-like surface structures. In addition, a layer of wax that exists on the epidermis of the cicada wing is a low surface tension material [25] and can serve naturally as an anti-sticking coating during the separation process of nanocauliflower arrays.
This is in contrast to the cases of artificial templates where the anti-sticking treatment has to be carried out by self-assembling a monolayer of fluorine or methyl-terminated organic molecules to avoid adherence [30,31].

The existence of abundant branches in the structure of cauliflower-like nanopatterns results in considerable optical properties, particularly in their reflection spectrum. These branches can cause to trap the light and, consequently, to reduce the reflectivity of the substrate in broadband wavelength ranges, although their aspect ratio (i.e. the height divided by the period) is not high. The diffused reflections of three samples fabricated using different operational conditions of sputtering $\left(\mathrm{C}_{2}, \mathrm{C}_{3}, \mathrm{C}_{4}\right)$ compared with the planar silver substrate are shown in Fig. 4. Sample $\mathrm{C}_{4}$ related to the mature silver nanocauliflower-like arrays indicates a reduced light reflection over all the visible spectra. This specific characteristic of branched noble metal nano structures can be important for the operation of organic solar cells to enhance their light harvesting and performance.

\section{B. Performance of OSCs with branched cauliflower-like nanopattern}

The branched cauliflower-like nanopattern was introduced into the planar device to improve its performance and photon absorption. The $\mathrm{J}-$ $\mathrm{V}$ characteristics of this OSC (compared to OSC with a planar back electrode) are shown in Fig. 5, and the detailed performance is summarized in Table 2. An increase of $14.8 \%$ in performance $(\mathrm{PCE}=1.95 \%$ and $\mathrm{PCE}=$ $2.24 \%$ for the planar and nanopatterned devices respectively) was achieved mainly due to the enhancement in the photo-generated current $\left(6.07 \mathrm{~mA} / \mathrm{cm}^{2}\right.$ and $7.11 \mathrm{~mA} / \mathrm{cm}^{2}$ for the planar device and the nanopatterned device respectively). This current density improvement is, in the first place, attributed to light scattering in the branched nanostructure, increasing the device optical thickness, and enhancing its light-harvesting efficiency. Fig. 6 presents the optical absorption spectra from the diffuse reflection $(\mathrm{R})$ and transmission $(\mathrm{T})$, 
using 1-R-T over the wavelength range of 350$850 \mathrm{~nm}$. The optical absorption demonstrated an increase of optical density for a branched structure introduced to the device when compared to the planar device. Over the visible range, an average absorption increase of $20 \%$ in the cauliflower-like nanopatterned device can be observed in comparison with the planar device. The absorption spectrum of the nanopatterned device exhibits maximum absorption in the range of 470-550 nm, which is a result of the interface nanostructure that is facilitated by excitation of surface modes. Also, the enhancement absorption calculated using dividing of the difference between absorption of the nanopatterned and planar devices by that of the planar device [21], can be found in a larger wavelength range between $700-850 \mathrm{~nm}$. These enhanced ranges of absorption spectrum are outside the bulk plasmon resonance of silver [32], but the strong surface plasmon resonance mode can be excited at longer wavelengths than those of bulk plasmon resonances [8,9].

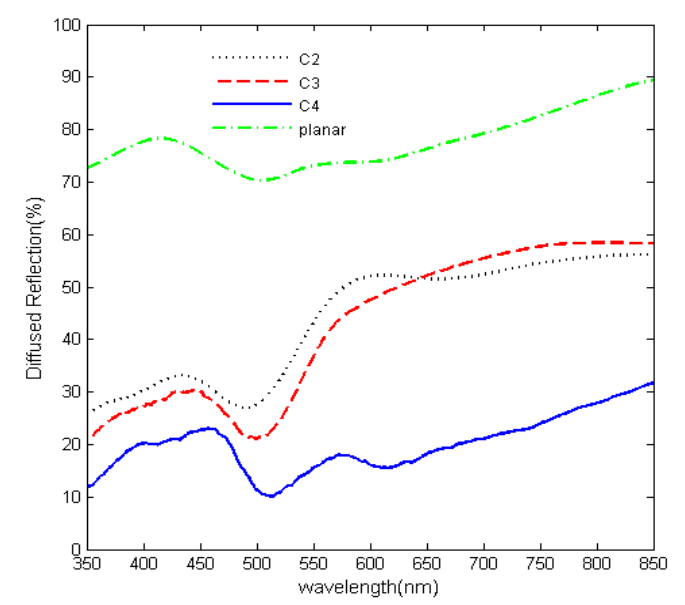

Fig. 4. Diffused reflection intensity of three nanostructure samples $\left(\mathrm{C}_{2}, \mathrm{C}_{3}\right.$, and $\mathrm{C}_{4}$ compared with the planar substrate) fabricated in different operational conditions of magnetron sputtering vs. incident light wavelength.

The first reason for improvement of light absorption stems from the especial geometry of the branched nanostructures on the back electrode. Thanks to the size of cauliflowerlike nanopatterns being in the range of the subwavelength of visible light; they serve as integrated scattering elements diffusing the reflected light. The light reflected by the branched nanostructures is no longer normal to the substrate plane. Thus, it crosses the absorber layer at a longer angle on the path way back and enhances the optical path length of the incident light, where eventually light absorption is improved in the active layer and the photo-generated current $[8,33,34]$.

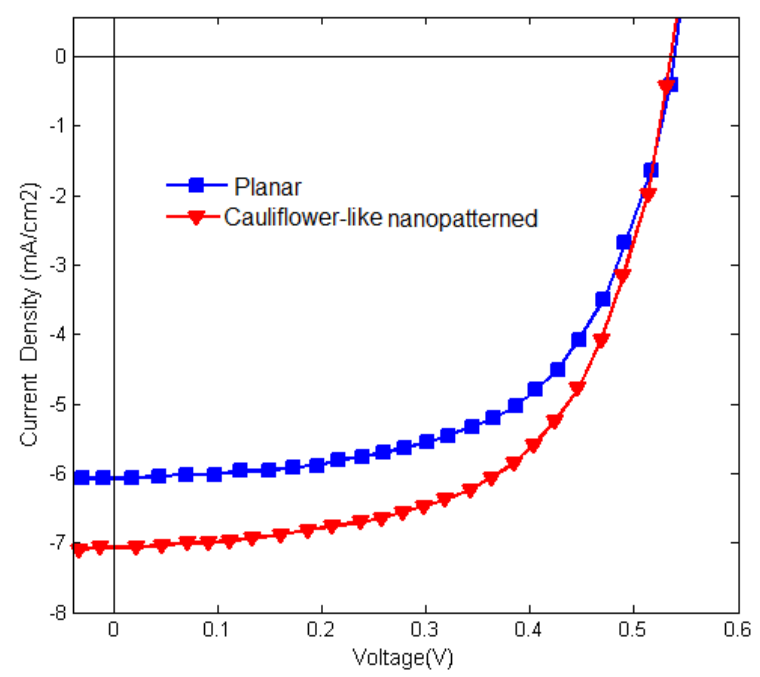

Fig. 5. Current density-voltage (J-V) characteristics of the planar and cauliflower-like nanopatterned back electrode devices measured under AM 1.5 illuminations at $100 \mathrm{~mW} / \mathrm{cm}^{2}$

Table 2. Photovoltaic parameters of the OSCs of the planar and nanopatterned cauliflower like back electrode under AM $1.5 \mathrm{G}$ illumination at 100 $\mathrm{mW} / \mathrm{cm}^{2}$.

\begin{tabular}{ccccc}
\hline \hline Device & $\begin{array}{l}\text { Voc } \\
(\mathrm{V})\end{array}$ & $\begin{array}{c}\text { Jsc } \\
\left(\mathrm{mA} / \mathrm{cm}^{2}\right)\end{array}$ & $\begin{array}{c}\text { FF } \\
(\%)\end{array}$ & $\begin{array}{c}\text { PCE } \\
(\%)\end{array}$ \\
\hdashline$-\mathrm{Clanar}^{2}$ & 0.54 & 6.07 & 0.58 & 1.95 \\
\hdashline Nanopatterned & 0.54 & 7.11 & 0.59 & 2.24 \\
\hline \hline
\end{tabular}

Physically, another reason of the enhanced absorption behavior stems from excitation of surface plasmons in silver nanocauliflowers. Due to the negative real part of the dielectric constant of noble metal materials, the incident light excites surface plasmon resonances (SPRs) [34-38]. These resonances can generally occur at discrete frequencies and are strongly localized in the skin surface layer of nanostructure arrays. Due to the dipole-dipole interaction between the ensemble of nanostructures and the strong coupling between localized plasmon, these resonances tend to broaden and produce tightly bound 
bands. Furthermore, the existence of plasmon bands is a necessary condition for creating plasmonic broadband absorber structures.

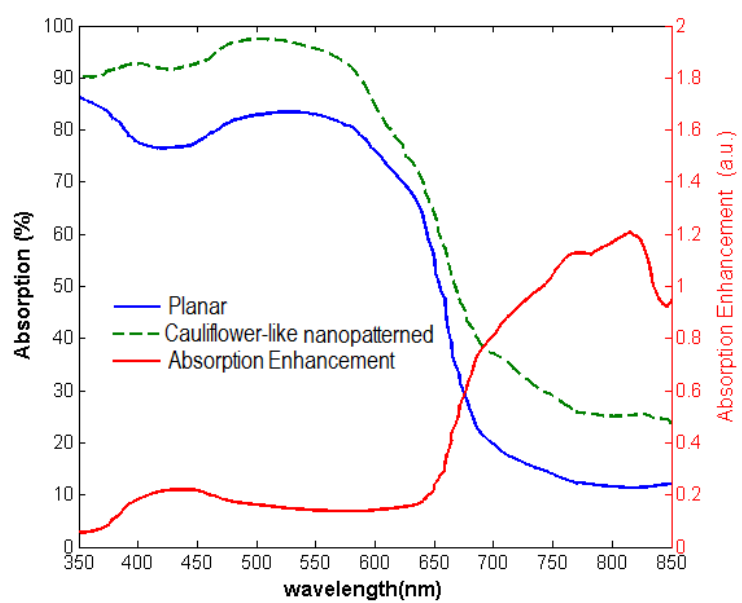

Fig. 6. Optical absorption spectra of the planar (solid) and cauliflower-like nanopatterned (dashed) solar cells.

By introducing these nanocauliflower arrays on the back electrode, as a consequence of the existence of abundant branches and gaps on their surface with dimensions near the organic exciton diffusion length, preferential carrier pathways and efficient capture and separation of excitons could be provided; thus reducing the charge recombination and facilitating the carrier collection in OSCs [10]. Also, formation of nanostructured metallic layers in the form of a deep diffraction grating nonresonant (broadband) absorption of unpolarized light has been also confirmed with plasmonic composite metamaterials [37]. Ag nanocauliflower arrays are comparable to subwavelength geometrically ordered metal arrays such as cylinders [38] and spheres [39] and have efficient absorption of visible light. However, in cylindrical and spherical structures, broadband absorption is comparatively inefficient for wavelengths longer than the SP resonance wavelength.

\section{Conclusion}

In this research, we demonstrated the fabrication of template-based branched cauliflower-like nanostructures of silver metal on bio-templates of cicada wing patterns and their application as a back electrode in organic solar cells. The self-assembled fabrication process was carried out using a gas aggregation nanocluster source through $\mathrm{dc}$ magnetron sputtering. A power conversion efficiency enhancement of $14.8 \%$ was achieved as compared to that of the planar device. Such a result originates from an increase in photo-generated current as a consequence of an average enhancement of $20 \%$ in optical absorption. The enhancement in the optical absorption resulting from the improvement of light trapping in the active layer stems from the especial geometry of the branched nanopatternes on the back electrode and the plasmonic behavior of silver as a noble metal material. The increased interfacial area induced by the branched cauliflower-like nanopatterns is also contributed to the increased efficiency by improving the capture of the excitons at the branched interfaces.

\section{ACKNOWLEDGMENTS}

The authors would like to thank Photonics Research Group of Yazd University (YPRG) for laboratory support. Also, this study was partly supported financially by the Ministry of Energy, Yazd Regional Branch.

\section{REFERENCES}

[1] M.-Y. Lin, Y. L. Kang, Y.-C. Chen, T.H. Tsai , S.-C. Lin, Y.-H. Huang, Y.-J. Chen, C.-Y. Lu, H. Y. Lin, L. A. Wang, C.-C. Wu, and S.C. Lee, "Plasmonic ITO-free polymer solar cell," Opt. Express, Vol. 22, pp. 438-445, 2014.

[2] Y. Yang, K. Lee, K. Mielczarek, W. Hu, and A. Zakhidov, "Nanoimprint of dehydrated PEDOT:PSS for organic photovoltaics," Nanotechnol. Vol. 22, pp. 485301 (1-9), 2011.

[3] C. Cocoyer, L. Rocha, L. Sicot, B. Geffroy, R. de Bettignies, C. Sentein, C. FioriniDebuisschert, and P. Raimond "Implementation of submicrometric periodic surface structures toward improvement of organic-solar-cell performances," Appl. Phys. Lett. Vol. 88, pp 133108 (1-8), 2006.

[4] S. Jeong, E. C. Garnett, S. Wang, Z. Yu, S. Fan, M. L. Brongersma, M. D. McGehee, and Y. Cui, "Hybrid silicon nanocone-polymer solar cells," Nano Lett. Vol. 12, pp. 29712976, 2012. 
[5] D.-H. Ko, J. R. Tumbleston, L. Zhang, S. Williams, J. M. DeSimone, R. Lopez, and E. T. Samulski, , "Photonic crystal geometry for organic solar cells," Nano Lett. Vol. 9, pp. 2742-2746, 2009.

[6] F. C. J. Wu, Y. Hsiao, F. Chien, P. Chen, C. Kuo, M. Huang, and C. Hsu, "Surface plasmonic effects of metallic nanoparticles on the performance of polymer bulk heterojunction solar cells," ACS Nano, Vol. 5, pp. 959 (1-9), 2011.

[7] J. N. Munday and H. A. Atwater, "Large Integrated Absorption Enhancement in Plasmonic Solar Cells by Combining Metallic Gratings and Antireflection Coatings," Nano Lett. Vol. 11, pp. 2195-2201, 2010.

[8] R. B. Dunbar, H. C. Hesse, D. S. Lembke, and L. Schmidt-Mende, "Light-trapping Plasmonic nanovoid array," Physical Review B Vol. 85, pp. 035301 (1-7), 2012.

[9] H. A. Atwater and A. Polman, "Plasmonics for improved photovoltaic devices," Nature Mater. Vol. 9, pp. 205-213, 2010.

[10] A. Ghadirzadeh, L. Passoni, G. Grancini, G. Terraneo, A. Li Bassi, A. Petrozza, and F. Di Fonzo, "Hyperbranched Quasi-1D $\quad \mathrm{TiO}_{2}$ Nanostructure for Hybrid Organic-Inorganic Solar Cells," ACS Appl. Mater. Interfaces, Vol. 7, pp. 7451-7455, 2015.

[11] I. Gur, N. A. Fromer, C. -P. Chen, A. G. Kanaras, and A. P. Alivisatos, "Hybrid Solar Cells with Prescribed Nanoscale Morphologies Based on Hyperbranched Semiconductor Nanocrystals," Nano Lett. Vol. 7, pp. 409-414, 2007.

[12] W. Chen, M. P. Nikiforov, and S. B. Darling, "Morphology Characterization in Organic and Hybrid Solar Cells," Energy Environment. Sciences, Vol. 5, pp. 8045-8074, 2012.

[13] B. Q. Sun, E. Marx, and N. C. Greenham, "Photovoltaic devices using blends of branched CdSe nanoparticles and conjugated polymers," Nano Lett. Vol. 3, pp. 961-963, 2003.

[14] C. J. DeSantis and S. E. Skrabalak, "Core values: elucidating the role of seed structure in the synthesis of symmetrically branched nanocrystals," J. Amer. Chem. Soc. Vol. 135, pp. 10-13, 2012.

[15] J. Gong, G. Li, and Z. Tang, "Self-assembly of noble metal nanocrystals: fabrication, optical property, and application," Nano Today Vol. 7, pp. 564-585 2012.

[16] K. Jasuja and V. Berry, "Implantation and growth of dendritic gold nanostructures on graphene derivatives: electrical property tailoring and Raman enhancement," ACS Nano, Vol. 3, pp. 2358-2366, 2009.

[17] S. Y. Li and M. Wang, "Branched metal nanoparticles: a review on wet-chemical synthesis and biomedical applications," Nano Life, Vol. 2, pp. 1230002 (1-22), 2012.

[18] M. Shekhar, J. Wang, W.-S. Lee, W. D. Williams, S. M. Kim, E.A. Stach, J. T. Miller, W. N. Delgass, and F. H. Ribeiro, "Size and support effects for the water-gas shift catalysis over gold nanoparticles supported on model $\mathrm{Al}_{2} \mathrm{O}_{3}$ and $\mathrm{TiO}_{2}$," J. Amer. Chem. Soc., Vol. 134, pp. 4700-4708, 2012.

[19] L. Zhong, X. Zhai, X. Zhu, P. Yao, and M. Liu, "Vesicle-directed generation of gold nanoflowers by gemini amphiphiles and the spacer-controlled morphology and optical property," Langmuir, Vol. 26, pp. 5876-5881, 2009.

[20] C.-H. Sun, A. Gonzalez, N. C. Linn, P. Jiang, and B. Jiang, "Templated biomimetic multifunctional coatings," Appl. Phys. Letters, Vol. 92, pp. 051107 (1-4), 2008.

[21] J. You, X. Li, F.-X. Xie, W. E. I. Sha, J.H. W. Kwong, G. Li, W. C. H. Choy, and Y. Yang , "Surface Plasmon and Scattering-Enhanced Low-Bandgap Polymer Solar Cell by a Metal Grating Back Electrode," Adv. Energy Mater. Vol. 2, pp. 1203-1207, 2012.

[22] G. Xie, G. Zhang, F. Lin, J. Zhang, Z. Liu, and $\mathrm{S} . \mathrm{Mu}$, "The fabrication of subwavelength antireflective nanostructures using a biotemplate," Nanotechnol. Vol. 19, pp. 095605 (1-6), 2008.

[23] S. -H. Hong, J. Hwang, and H. Lee, "Replication of cicada wing's nano-patterns by hot embossing and UV nanoimprinting," Nanotechnol. Vol. 20, pp. 285303 (1-5), 2009.

[24] Y.-F. Huang, Y.-J. Jen, L.-C. Chen, K.-H. Chen, and S. Chattopadhyay, "Design for Approaching Cicada-wing Reflectance in Low and High Index Biomimetic Nanostructures," ACS Nano, Vol. 9, pp. 301-311, 2015.

[25] J. F. Vincent and U. G. Wegst, "Design and mechanical properties of insect cuticle," 
Arthropod Structure and Development, Vol. 33, pp. 187-199, 2004.

[26] H. Nourolahi, M. A, Bolorizadeh, and A. Behjat, "Light absorption with branched gold cauliflower-like nanostructure arrays," Vacuum, Vol. 123, pp. 29-34, 2015.

[27] Y. Guyot, B. Champagnon, M. Boudeulle, P. Mélinonb, B. Prévelb, V. Dupuisb, and A. Perezb, "Luminescence and Raman characterization of molecular and nanocrystalline silicon clusters," Thin Solid Films, Vol. 297, pp. 188-191, 1997.

[28] R. Hippler, S. Wrehde, V. Straňák, O. Zhigalov, H. Steffen, M. Tichý, M. Quaas, and $\mathrm{H}$. Wulff, "Characterization of a magnetron plasma for deposition of titanium oxide and titanium nitride films," Contributions to Plasma Phys. Vol. 45, pp. 348-357, 2005.

[29] M. Ganeva, T. Peter, S. Bornholdt, H. Kersten, T. Strunskus, V. Zaporojtchenko, F. Faupel, and R. Hippler, "Mass Spectrometric Investigations of Nano-Size Cluster Ions Produced by High Pressure Magnetron Sputtering," Contributions to Plasma Phys. Vol. 52, pp. 881-889, 2012.

[30] T. Bailey, B.J. Choi, M. Colburn, M. Meissl, S. Shaya, J. G. Ekerdt, S. V. Sreenivasan, and C. G. Willson, "Step and flash imprint lithography: Template surface treatment and defect analysis," J. Vacuum Science Technol. B, Vol. 18, pp. 3572-3577, 2000.

[31] M. Beck, M. Graczyk, I. Maximova, E.-L. Sarwe, T.G.I. Ling, M. Keilb, and L. Montelius, "Improving stamps for $10 \mathrm{~nm}$ level wafer scale nanoimprint lithography," Microelectron. Eng. Vol. 61-62, pp. 441-448, 2002.

[32] A. Liebsch, "Surface plasmon dispersion of Ag," Phys. Rev. Lett. Vol. 71, pp. 145-148, 1993.

[33] A. Baba, N. Aoki, K. Shinbo, K. Kato, and F. Kaneko, "Grating-coupled surface plasmon enhanced short-circuit current in organic thinfilm photovoltaic cells," ACS Appl. Mater. Interfaces, Vol. 3, pp. 2080-2084, 2011.

[34] D. Gollmer, F. Walter, C. Lorch, J. Novák, b. R. Banerjee, J. Dieterle, G. Santoroc, F. Schreiber, D.P. Kern, and M. Fleischer, "Fabrication and characterization of combined metallic nanogratings and ITO electrodes for organic photovoltaic cells," Microelectron. Eng. Vol. 119, pp. 122-126, 2014.

[35]F. G. De Abajo, "Colloquium: Light scattering by particle and hole arrays," Rev. Modern Phys. Vol. 79, pp. 1267 1290, 2007.

[36] T. Søndergaard, S. M. Novikov, T. Holmgaard, R. L. Eriksen, J. Beermann, Z. Han, K. Pedersen, and S. I. Bozhevolnyi, "Plasmonic black gold by adiabatic nanofocusing and absorption of light in ultrasharp convex grooves," Nature Commun. Vol. 3, pp. 969 (1-6), 2012.

[37] V. Kravets, F. Schedin, and A. Grigorenko, "Plasmonic blackbody: Almost complete absorption of light in nanostructured metallic coatings," Phys. Rev. B, Vol. 78, pp. 205405 (1-10), 2008.

[38] A. Aubry, D. Y. Lei, A. I. FernándezDomínguez, Y. Sonnefraud, S. A. Maier, and J. B. Pendry, "Plasmonic light-harvesting devices over the whole visible spectrum," Nano Lett. Vol. 10, pp. 2574-2579, 2010.

[39] A. Fernández-Domínguez, S. Maier, and J. Pendry, "Collection and concentration of light by touching spheres: a transformation optics approach," Phys. Rev. Lett. Vol. 105, pp. 266807 (1-4), 2010.

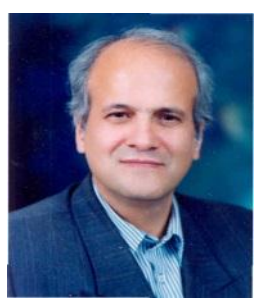

Mohammad Agha Bolorizadeh earned his B.Sc. in Physics from Shiraz University, Shiraz, Iran. Later, he earned his M.Sc. and $\mathrm{PhD}$ in atomic and molecular physics from University of Nebraska-Lincoln, Lincoln, Nebraska in 1979 and 1984, respectively.

He started his career at the Shahid Bahonar University of Kerman, Kerman, Iran as assistant professor in 1984. He later (in 2009) joined the Graduate University of Advanced Technology (GUAT), Mahan, Kerman, Iran. He had joined with the Atomic and Molecular Group at the Queen's University of Belfast in 1992 on a sabbatical leave. He also spent two 
sabbatical leaves at the Flinders University of South Australia, Adelaide, SA, Australia on 1997 and 2005. His research activities are on scattering of electron, photon and ions with atomic and molecular species and the related topics in science and technology. $\mathrm{He}$ established a reference laboratory to test fibers for communication use. His research, also, involves different aspects of photonic crystals and holly fibers to be used as sensors in the medical applications. He joined with the Physics Department at the University of Western Ontario, London, Ontario, Canada in 2000 as a visiting professor, where he worked on the ultra-shallow implantation of phosphorous on silicon.. $\mathrm{He}$ was also recognized as the Veteran of OPSI in January 2010.

Prof. Bolorizadeh is a member of the Optical Society of America, a member of Iranian Physical Society and a member of the Optics and Photonics Society of Iran (OPSI). He also served as the Head of the Organizing Committee of OPSI for 3 years starting from September 2010. He is currently directing the Atomic, Molecular and Oprical Section of the Iranian Physical Society.

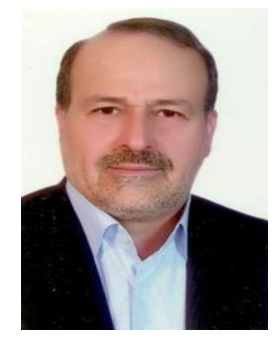

Abbas Behjat is the Professor of Physics, at Yazd University, Iran. He obtained his Ph.D. in x-ray lasers from the Essex University (UK) in 1996. Currently, he is group leader in the Photonics Research Group (PRG), Engineering Research Center, at Yazd University. Since 2007, he has been mainly involved in nanostructure-based optical devices including light emitting diodes, quantum dots and dye sensitized solar cells and also polymer bulk heterojunction solar cells.

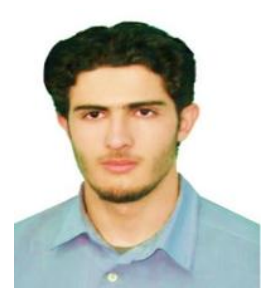

Hamzeh Nourolahi received his M.Sc. degree in Photonics from the Graduate University of Advanced Technology (Kerman), Iran in 2012. $\mathrm{He}$ is pursuing his Ph.D in Photonics at Graduate University of Advanced Technology cooperating with the Photonics Research Group at Yazd University under the supervision of Prof. Bolorizadeh and Prof. Behjat. His research focuses on the interactions of plasmons with third generation solar cells materials including Organic semiconductors and perovskites solar cells. 